\title{
Experimental and $a b$ initio molecular dynamics study of the structure and physical properties of liquid GeTe
}

\author{
Hans Weber, ${ }^{1,2}$ Mathias Schumacher, ${ }^{3}$ Pál Jóvári, ${ }^{4}$ Yoshimi Tsuchiya, ${ }^{5}$ Werner Skrotzki, ${ }^{2}$ \\ Riccardo Mazzarello, ${ }^{3,6, *}$ and Ivan Kaban ${ }^{1, \dagger}$ \\ ${ }^{1}$ IFW Dresden, Institute for Complex Materials, Helmholtzstrasse 20, 01069 Dresden, Germany \\ ${ }^{2}$ Technische Universität Dresden, Institute of Structural Physics, 01062 Dresden, Germany \\ ${ }^{3}$ RWTH Aachen University, Institute for Theoretical Solid State Physics, 52056 Aachen, Germany \\ ${ }^{4}$ Wigner Research Centre for Physics, Institute for Solid State Physics and Optics, P.O. Box 49, 1525 Budapest, Hungary \\ ${ }^{5}$ Niigata University, Faculty of Science, Department of Physics, Ikarashi 2-8050, Niigata 950-21, Japan \\ ${ }^{6}$ JARA-FIT and JARA-HPC, RWTH Aachen University, 52056 Aachen, Germany \\ (Received 14 April 2017; revised manuscript received 16 July 2017; published 10 August 2017)
}

\begin{abstract}
GeTe is a prototypical phase-change material employed in data storage devices. In this work, the atomic structure of liquid GeTe is studied by x-ray and neutron diffraction in the temperature range from 1197 to $998 \mathrm{~K}$. The dynamic viscosity is measured from 1273 to $953 \mathrm{~K}$, which is $55 \mathrm{~K}$ below the solidification point, using an oscillating-cup viscometer. The density of liquid GeTe between 1293 and $973 \mathrm{~K}$ is determined by the high-energy $\gamma$-ray attenuation method. The experiments are complemented with ab initio molecular dynamics (AIMD) simulations based on density functional theory (DFT). Compatibility of the AIMD-DFT models with the diffraction data is proven by simultaneous fitting of all data sets in the frame of the reverse Monte Carlo simulation technique. It is shown that octahedral order dominates in liquid $\mathrm{GeTe}$, although tetrahedral structures are also present. The viscosity of the equilibrium and weakly undercooled liquid GeTe obeys the Arrhenius law with a small activation energy of the order of $0.3 \mathrm{eV}$, which is indicative of a highly fragile liquid. The calculated density of states and electronic wave functions point to the existence of a pseudogap and localized electron states within the gap in the equilibrium liquid near the melting point as well as in the undercooled liquid.
\end{abstract}

DOI: 10.1103/PhysRevB.96.054204

\section{INTRODUCTION}

Chalcogenide alloys along the GeTe- $\mathrm{Sb}_{2} \mathrm{Te}_{3}$ pseudobinary line have attracted considerable attention for several decades due to the phase-change behavior and the remarkable differences in physical properties between the crystalline and the amorphous phases [1-5]. The optical reflectivity contrast is widely exploited in optical data storage media such as rewritable CDs, DVDs, and Blu-ray Discs. The electrical resistivity difference is of particular interest for the nonvolatile phase-change memory (PCM). It is expected that PCM will outperform the existing nonvolatile memory technologies, e.g., flash memory [6].

The functioning of the PCM is based on the switching of an active material from the amorphous to the crystalline state in a set operation and from the crystalline to the amorphous state in a reset operation, thereby passing either via the supercooled liquid state (devitrification) or via the liquid state (vitrification). This implies that the performance of the PCMs is to a large extent determined by the atomic structure and dynamics of the phase-change material in the liquid and supercooled liquid state. Therefore, investigations of the dynamic and structural properties of these states are of high importance for the development of highly efficient memories.

GeTe is a base alloy for the Ge-Sb-Te family and a prototypical phase-change alloy [7], which therefore has been thoroughly studied in the crystalline [8-11] and amorphous

\footnotetext{
*mazzarello@physik.rwth-aachen.de

†i.kaban@ifw-dresden.de
}

states [7,12-16]. However, it has been poorly studied in the liquid and supercooled liquid states. The experimental data exist only for the electrical resistivity [17], the sound velocity, and the adiabatic compressibility [18] of liquid GeTe, determined in a relatively wide temperature range above the melting point. As regards kinetic properties, we are aware of only one paper on the dynamic viscosity of liquid GeTe [19], measured at two temperatures (1000 and $1025 \mathrm{~K})$ close to the melting point $T_{\mathrm{m}}=998 \mathrm{~K}$.

$A b$ initio molecular dynamics (AIMD) simulations based on density functional theory (DFT) have partly elucidated the structure and the bonding properties of liquid GeTe. Raty et al. [20] have shown that liquid GeTe is characterized by alternating short and long bonds indicative of a Peierls distortion [21] close to the melting temperature, which disappears slowly upon heating. This finding has been rationalized in terms of a gradual transition from a semiconducting to a metallic liquid. The chemical order in liquid GeTe has revealed interesting features, too. In particular, a large number of homonuclear $\mathrm{Ge}$ Ge and Te-Te bonds, with coordination numbers $N_{\mathrm{GeGe}} \approx 2.56$ and $N_{\mathrm{TeTe}} \approx 1.11$, have been observed in the DFT model of liquid GeTe at $1000 \mathrm{~K}$ [20]. The homonuclear bonds have also been found in another DFT model of liquid GeTe [22], but the respective coordination numbers were smaller $\left(N_{\mathrm{GeGe}} \approx 1.7\right.$ and $N_{\mathrm{TeTe}} \approx 0.7$ ). Ge-Ge bonds have been shown to be present in rapidly quenched amorphous $\mathrm{GeTe}$, resulting in a significant number of tetrahedrally coordinated Ge atoms in this phase [13-15].

The aim of the present study is to gain a comprehensive understanding of the structural and dynamic properties of the GeTe alloy in the liquid and supercooled liquid state using a combined experimental and theoretical approach. For 
this purpose, $\mathrm{x}$-ray and neutron diffraction experiments, and density and viscosity measurements have been performed. As far as DFT simulations are concerned, the van der Waals functional developed by Lee et al. [23] (denoted as vdW-DF2), which has recently been shown to yield accurate structural models of amorphous GeTe [13] and liquid $\mathrm{Ge}_{2} \mathrm{Sb}_{2} \mathrm{Te}_{5}$ [24], is employed in the present work. Analysis of the chemical and topological short-range atomic order, and the dynamic and electronic properties extracted from the AIMD models provide a complete picture of the GeTe alloy in the liquid and weakly supercooled liquid states.

\section{METHODS}

\section{A. Experiment}

GeTe master alloys are prepared from Ge and Te of high purity (both $99.999 \%$ ). The proper quantities of $\mathrm{Ge}$ and $\mathrm{Te}$ are loaded into quartz ampoules, which subsequently are evacuated, filled with Ar to a pressure of about 250 mbar at room temperature, and sealed. The ampoules are heated to $1073 \mathrm{~K}$ at a rate of $5 \mathrm{~K} \mathrm{~min}^{-1}$, held for $5 \mathrm{~h}$ to assure homogenization of the alloys, and slowly cooled to room temperature.

A series of x-ray diffraction (XRD) measurements of liquid GeTe is carried out at the beamline ID11 at the European Synchrotron Radiation Facility (ESRF, Grenoble). Heating of the sample is done with a hot-air blower commercialized by Cyberstar (France) and provided by ESRF. Pieces of a master alloy are loaded into a quartz-glass capillary ( $2 \mathrm{~mm}$ diameter and $0.05 \mathrm{~mm}$ wall thickness), which is then evacuated and filled with Ar at about 250 mbar. Afterwards, GeTe pieces are molten using a gas burner in order to get a homogeneous sample of about $5 \mathrm{~mm}$ height in the capillary. The capillary with the sample is set into a thin-walled stainless-steel holder of $2.5 \mathrm{~mm}$ inner diameter, which is concentrically mounted in a ceramic tube of $6 \mathrm{~mm}$ inner diameter, placed just above the nozzle of the hot-air blower. The hot air is directed into the space between the steel and ceramic tubes and efficiently heats the sample. There are two rectangular windows $\left(1.5 \times 2.5 \mathrm{~mm}^{2}\right)$ on opposite sides of the tubes for the incident and diffracted $\mathrm{x}$-ray beam. The centers of these windows as well as the middle of the sample are aligned with the incident beam axis. The temperature of the hot-air blower is controlled by a Eurotherm regulator with a precision of $\pm 1 \mathrm{~K}$. The temperature of the sample is calibrated using a type $\mathrm{K}$ thermocouple placed in a quartz capillary at the sample position in a dedicated heatingcooling run. The XRD experiments at ID11 are performed with a photon energy of $120 \mathrm{keV}$ and a beam size of $0.5 \times 0.5 \mathrm{~mm}^{2}$ in transmission geometry. The scattering intensity is collected by a FReLoN charge-coupled device (CCD) camera during heating the liquid GeTe sample up to $1077 \mathrm{~K}$ and subsequent cooling into the solid state. The accessed upper limit of the wave vector $Q$ is about $18 \AA^{-1}$.

XRD measurements up to a higher temperature of $1197 \mathrm{~K}$ are carried out at the P07 experimental station at the Synchrotron Light Source PETRA III (DESY, Hamburg). In this case, GeTe alloy sealed in a thin-walled quartz-glass capillary as described above, is heated by an induction heater in an electromagnetic levitation facility [25]. The temperature is measured with a type $\mathrm{K}$ thermocouple touching the bottom of the capillary as well as by an optical pyrometer from the top. The pyrometer is calibrated using the melting temperature of GeTe. The energy of the photon beam is as high as $121.3 \mathrm{keV}$ and the beam size is $0.5 \times 0.5 \mathrm{~mm}^{2}$. Due to the peculiarities of the setup, the upper limit of the wave vector is about $12 \AA^{-1}$.

The two-dimensional XRD patterns measured at ESRF are radially integrated using the PYFAI package [26] and those measured at DESY are integrated using the FIT2D program [27]. In both series of the XRD measurements, the sample-to-detector distance and the orthogonality of the detector with respect to the incident beam are calibrated using $\mathrm{CeO}_{2}$. The integrated intensities are corrected for background, absorption, and incoherent scattering. The latter is done using the analytic approximations of Balyuzi [28]. The corrected XRD intensities are normalized to the Faber-Ziman [29] total structure factors using X-ray atomic form factors from Waasmaier and Kirfel [30].

The neutron diffraction (ND) measurements are carried out at the 7C2 diffractometer of the ORPHÉE reactor, Laboratoire Léon Brillouin (LLB), CEA-CRNC Saclay, France. A GeTe sample is contained in a quartz-glass ampoule $(8 \mathrm{~mm}$ inner diameter, $1 \mathrm{~mm}$ wall thickness, $55 \mathrm{~mm}$ sample height), which has been vacuumed and sealed under Ar. The ampoule with the sample is placed into a vanadium furnace controlled by a Eurotherm regulator. The temperature is measured with two type $\mathrm{K}$ thermocouples touching the ampoule walls. The wavelength of the incident radiation is $0.72 \AA$ and the beam size $12 \times 45 \mathrm{~mm}^{2}$. The scattered intensity is measured by a ${ }^{3} \mathrm{He}$ position sensitive detector and converted to $I(2 \theta)$ using the program SCRIPT provided by LLB. The measured data are corrected for detector efficiency, empty instrument background, scattering from the furnace, and the sample container, multiple scattering [31], and absorption [32]. The Faber-Ziman total structure factors are calculated from corrected intensities using the neutron scattering lengths from Sears [33].

The density of liquid and slightly supercooled liquid GeTe is determined by the high-energy $\gamma$-ray attenuation method, giving an accuracy better than $\pm 0.5 \%$ as described in Ref. [34]. About $110 \mathrm{MBq}$ of ${ }^{137} \mathrm{Cs}$ is used as a $\gamma$-ray source of $662 \mathrm{keV}$ and a NaI scintillation counter is used as the $\gamma$-ray detector. The GeTe sample is sealed in a quartz ampoule with a diameter of $1.1 \mathrm{~cm}$ and a length of $2.388 \pm 0.002 \mathrm{~cm}$. The latter is determined by measuring the linear attenuation coefficient of $\mathrm{Hg}$ at room temperature. The linear attenuation coefficients of pure Ge $\left[(7.005 \pm 0.004) \times 10^{-3} \mathrm{~m}^{2} \mathrm{~kg}^{-1}\right]$ and Te $[(7.182 \pm$ $\left.0.003) \times 10^{-3} \mathrm{~m}^{2} \mathrm{~kg}^{-1}\right]$ are measured using freshly powdered specimens compressed into a cylindrical steel tube.

The dynamic viscosity of liquid GeTe is measured with an accuracy better than $10 \%$ by means of the oscillating-cup method as described elsewhere [35]. The sample is sealed in a quartz ampoule with $22.5 \mathrm{~mm}$ inner diameter under about 250 mbar Ar after previous vacuuming. A graphite container with the ampoule is connected to a torsion wire and placed in a high-vacuum vertical furnace. The temperature is measured with an accuracy better than $\pm 5 \mathrm{~K}$ by a thermocouple situated near the bottom of the sample container. The system is heated at a rate of $5 \mathrm{~K} \mathrm{~min}^{-1}$ to $1273 \mathrm{~K}$ and held for $1 \mathrm{~h}$ at constant temperature. Ten to twelve torsion oscillations are registered for each point measured during the cooling of the GeTe melt at a rate of $1 \mathrm{~K} \mathrm{~min}^{-1}$. The dynamic viscosity of the melt is 
calculated by simulating the measured damped oscillations using the equation given by Roscoe and Bainbridge [36] and modified by Brooks et al. [37].

\section{B. Modeling}

The AIMD simulations of liquid GeTe are carried out with the QUICKSTEP code included in the CP2K package [38]. This code uses a mixed Gaussian and plane-wave basis set. The Kohn-Sham orbitals are expanded in a Gaussian-type basis set of triple zeta plus polarization quality, whereas the charge density is expanded in plane waves, with a cutoff of 300 Ry. Scalar-relativistic Goedecker [39] pseudopotentials obtained from calculations based on the local density approximation (LDA) are used. The Brillouin zone is sampled only at the $\Gamma$ point. A very efficient AIMD method developed by Kühne et al. [40], intrinsically dissipative and combined with a Langevin thermostat to guarantee the simulations sample the canonical ensemble $[40,41]$, is used. It is important to mention that, in general, stochastic thermostats such as the Langevin thermostat affect the dynamical properties of a system significantly. The simulations of liquid GeTe are performed using cubic cells with 512 atoms. The dimensions of the cells and number densities are listed in Table S1 in the Supplemental Material [42]. The number densities are obtained by interpolation of the experimental values given in Table S2 and Fig. S1 in the Supplemental Material [42]. To generate a model, a random configuration is heated up to $3000 \mathrm{~K}$, kept at this high temperature for $30 \mathrm{ps}$, and subsequently equilibrated for $30 \mathrm{ps}$ at the respective target temperature. Starting from the equilibrated models, MD trajectories of up to 120 ps length are produced. The single MD steps are of 2 fs length each.

The self-diffusion coefficient $D$ of liquid GeTe is calculated from the mean square displacements (MSDs) of the particles using Einstein's formula,

$$
D=\frac{1}{6} \lim _{t \rightarrow \infty} \frac{\partial}{\partial t}\left\langle r^{2}(t)\right\rangle,
$$

where $\left\langle r^{2}(t)\right\rangle$ is the mean squared distance which the atoms cover in the time interval $t$. In practice, $D$ is calculated from the slope of the linear time dependence of the mean square displacement obtained from MD simulations.

The dynamic viscosity $\eta$ is determined from the diffusion coefficient $D$ using the Stokes-Einstein relation (SER),

$$
\eta=\frac{k_{B} T}{6 \pi R_{\text {hyd }} D} .
$$

Here, $k_{B}$ is the Boltzmann constant, $T$ is the absolute temperature, and $R_{\text {hyd }}=1.83 \AA$ is the effective hydrodynamic radius of GeTe taken from the work of Sosso et al. [43].

Reverse Monte Carlo simulations are performed using the $\mathrm{RMC}++$ code [44]. The cubic simulation boxes contain 20000 atoms with the same number densities as those used in the corresponding AIMD simulations. A starting atomic configuration is random distributions of atoms with the following minimum interatomic distances: $2.1 \AA$ for Ge-Ge, $2.2 \AA$ for Ge-Te, and $2.4 \AA$ for Te-Te. The atoms are moved randomly in order to converge the fit to the experimental XRD and ND structure factors and to all of the three partial pair distribution functions (PDFs) obtained from AIMD simulations.
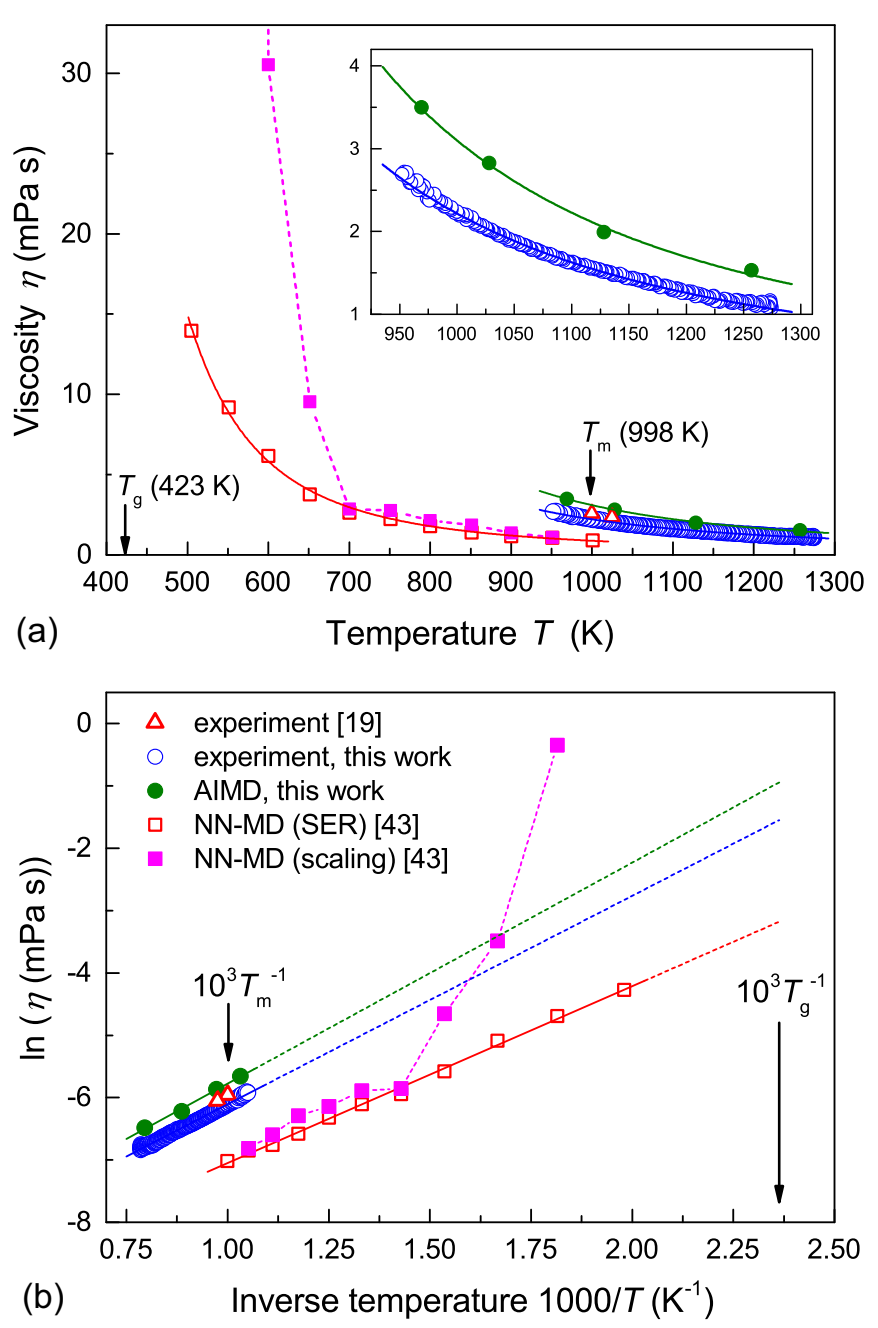

FIG. 1. Dynamic viscosity of liquid GeTe: (a) temperature dependence; (b) Arrhenius plot. Open blue circles: viscosity measured by oscillating-cup technique in the present work (the experimental uncertainty is comparable to the symbols' size); open red triangles: data obtained by the same method by Herwig and Wobst [19]; closed olive circles: values extracted from $a b$ initio MD simulations using the vdW-DF2 exchange-correlation functional in the present work; open and closed squares: values from MD simulations based on a neural-network potential approach, performed by Sosso et al. [43]; SER: viscosity calculated from the diffusion coefficient of a fixed-size cell and application of the Stokes-Einstein relation; scaling: viscosity computed by scaling the diffusion coefficient with the size of the simulation cell; full lines: fits of the viscosity data with the Arrhenius equation $\eta=\eta_{0} \exp \left(\frac{E_{a}}{k_{B} T}\right)$ (see main text for the resulting values of $\eta_{0}$ and $E_{a}$ ); dotted lines: guides for the eyes; black arrows indicate the glass transition and the melting temperature $\left(T_{g}=423 \mathrm{~K}\right.$ [53], $\left.T_{\mathrm{m}}=998 \mathrm{~K}\right)$.

\section{RESULTS AND DISCUSSION}

The measured dynamic viscosity of liquid GeTe is plotted in Fig. 1, whereas the tabulated values are given in Table S3 in the Supplemental Material [42]. Interestingly, it was possible to supercool the liquid GeTe by about $50 \mathrm{~K}$ under the melting point, whereas, in our previous study [24], the $\mathrm{Ge}_{2} \mathrm{Sb}_{2} \mathrm{Te}_{5}$ melt crystallized during the viscosity measurements at the same conditions without reaching the supercooled state. Similarly 


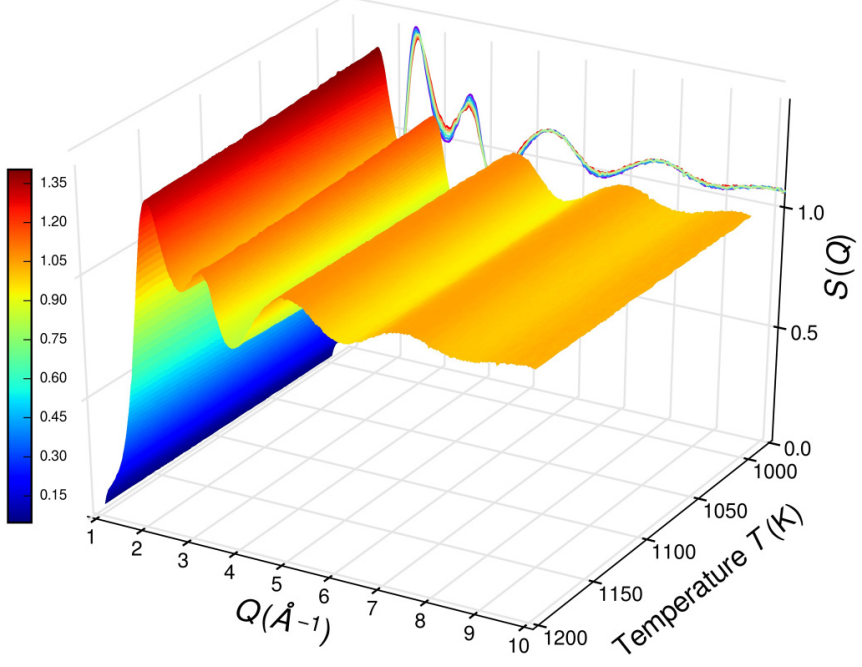

FIG. 2. XRD total structure factors $S(Q)$ for liquid GeTe measured upon cooling from 1197 to $998 \mathrm{~K}$. The family of curves projected to the rear wall of the graph provides an overview of the changes of $S(Q)$ with temperature.

to $\mathrm{Ge}_{2} \mathrm{Sb}_{2} \mathrm{Te}_{5}$ (experiment and AIMD simulations [24]), the dynamic viscosity of GeTe exhibits Arrhenius behavior in the equilibrium as well as in the weakly supercooled liquid state. Fitting the experimental values with the function

$$
\eta=\eta_{0} \exp \left(\frac{E_{a}}{k_{B} T}\right)
$$

yields the activation energy for the viscous flow $E_{a}=$ $0.293 \mathrm{eV}$ and a constant $\eta_{0}=0.074 \mathrm{mPa}$ s representing the viscosity in the limit of infinite temperature.

The Arrhenius-type dynamic viscosity of liquid GeTe correlates with the linear temperature dependences of other physical properties such as the sound velocity, adiabatic compressibility [18], and molar volume measured in this work (Fig. S1 and Table S2 in the Supplemental Material [42]). The total structure factors measured over the temperature range of about $200 \mathrm{~K}$ also show a continuous and linear evolution, as can be seen in Fig. 2. The positions and the heights of the peaks of the structure factors and of the pair distribution functions change linearly and rather weakly with temperature (Fig. 3). Small discrepancies observed in $r_{1}$ and $g\left(r_{1}\right)$ from the functions obtained by the two XRD measurements are mainly due to the different upper integration limits $Q_{\max }$ [45]. The largest differences of $0.5 \%$ for $r_{1}$ and $3.5 \%$ for $g\left(r_{1}\right)$ can be considered as uncertainties for these structural parameters.

To understand the atomic structure of a liquid alloy it is necessary to analyze the partial pair distribution functions $g_{i j}(r)$. As it is not possible to determine a set of three distributions from only two experimental data sets on liquid GeTe (XRD and ND), we compute them by AIMD modeling from 20-ps-long trajectories. Plausibility of the models is ensured by a simultaneous fit to the experimental XRD (measured up to $Q_{\max } \approx 18 \AA^{-1}$ ) and ND structure factors, and the AIMD partial pair distribution functions in the frame of the reverse Monte Carlo simulation technique (Fig. 4). The AIMD-simulated partial pair distribution functions $g_{i j}(r)$ as
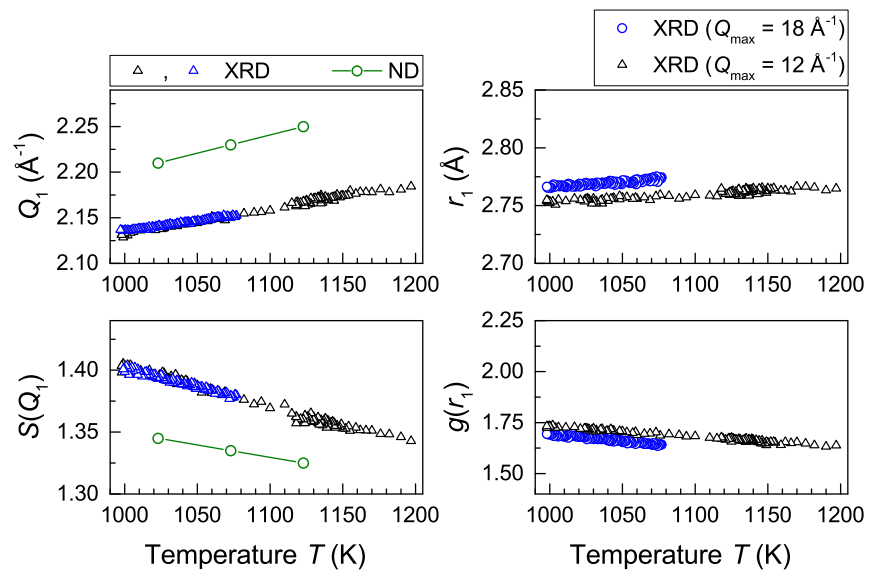

FIG. 3. Temperature dependence of the positions and the heights of the first peaks on the structure factors $\left[Q_{1}\right.$ and $\left.S\left(Q_{1}\right)\right]$ and on the pair distribution functions $\left[r_{1}\right.$ and $\left.g\left(r_{1}\right)\right]$ for liquid GeTe. The differences observed for $r_{1}$ and $g\left(r_{1}\right)$ from two XRD measurements are mainly due to different upper limits of the wave vector $Q_{\max }$.

well as the corresponding radial distribution functions (RDFs) $4 \pi r^{2} \rho_{0} g_{i j}(r)$ are plotted in Fig. 5. The respective total pair distribution functions and structure factors are shown along with the same curves obtained by x-ray diffraction in Fig. 6, also proving a good agreement between the simulations and experiment.

A partial PDF $g_{i j}(r)$ indicates the probability of finding an atom of sort $j$ at a distance $r$ from an atom of sort $i$. The most probable bond length $r_{i j}$ is determined by the position of the first maximum of $g_{i j}(r)$ and the coordination number $N_{i j}$ is calculated by integration of the radial distribution function over the first coordination shell:

$$
N_{i j}=\int_{R_{1}}^{R_{2}} 4 \pi r^{2} c_{i} \rho_{0} g_{i j}(r) d r .
$$
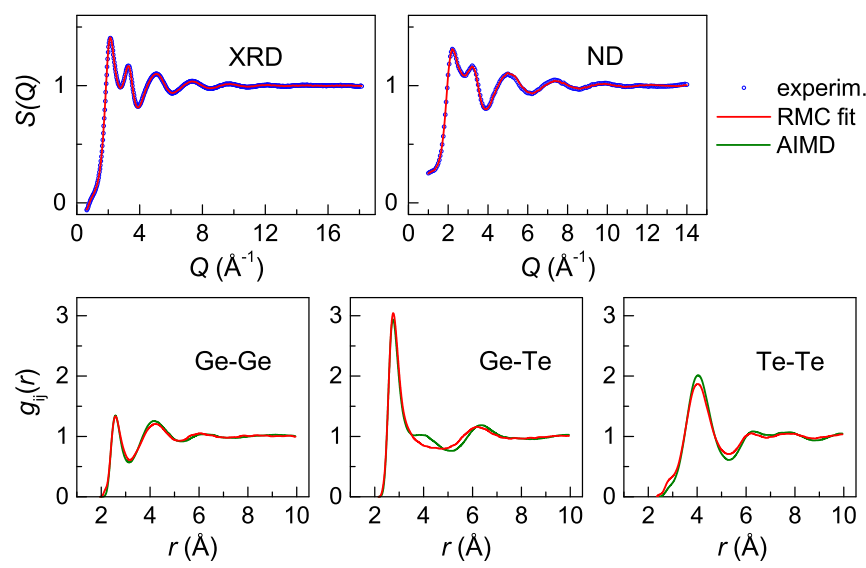

FIG. 4. RMC fits (red curves) obtained by simultaneous modeling of the x-ray and neutron diffraction total structure factors $S(Q)$ of liquid GeTe measured at $1023 \mathrm{~K}$ (open circles, blue) and partial pair distribution functions $g_{i j}(r)$ obtained from AIMD simulation at the effective temperature of $1032 \mathrm{~K}$ (olive curves). 

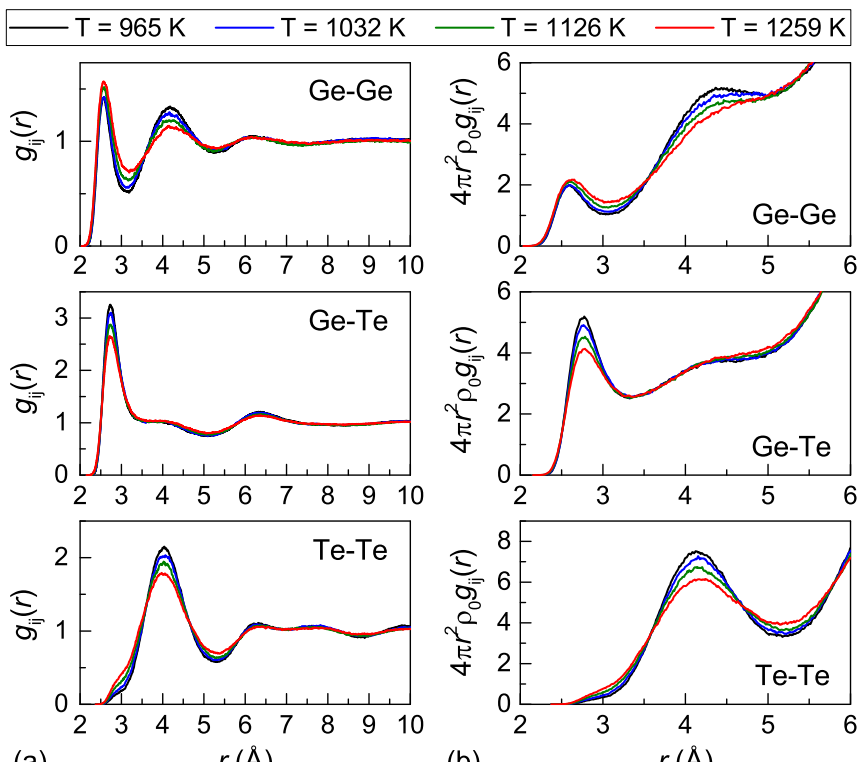

(b)

$r(\AA)$

FIG. 5. (a) Partial pair distribution functions $g_{i j}(r)$ and (b) radial distribution functions $4 \pi r^{2} \rho_{0} g_{i j}(r)$ for GeTe in the supercooled $(965 \mathrm{~K})$ and equilibrium liquid state $(1032,1126,1259 \mathrm{~K})$ obtained from AIMD simulations.

Here, $R_{1}$ and $R_{2}$ are the radial boundaries of the coordination shell, $c_{i}$ is the mole fraction of component $i$, and $\rho_{0}$ is the number density of the alloy.

The calculated partial $N_{i j}$ and total $N$ coordination numbers for liquid GeTe as well as the applied integration limits are given in Table I. $N_{\mathrm{GeGe}}$ and $N_{\mathrm{GeTe}}$ are obtained by integrating up to the first minimum appearing on the corresponding $g_{i j}(r)$ function. As can be seen in Fig. 5, the Te-Te distribution does not exhibit any peak in the range of $r$ values where it can be expected based on the studies of pure Te [45] and Te-rich Ge-Te melts [46], i.e., at about 2.8-2.9 $\AA$. However, there is a shoulder in $g_{\mathrm{TeTe}}(r)$ in this region, indicating the presence of Te-Te bonds in liquid GeTe. To estimate $N_{\text {TeTe }}$, a cutoff of $3.4 \AA$
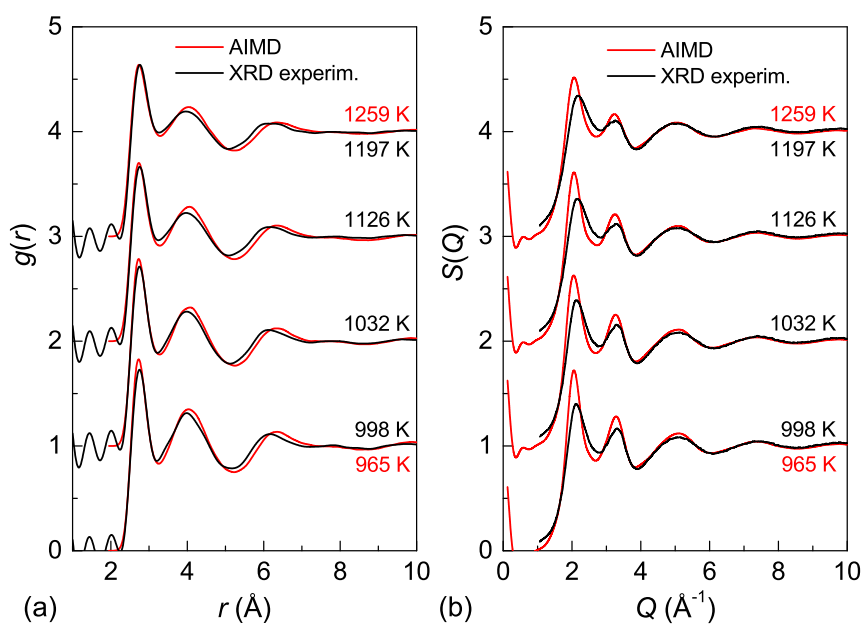

FIG. 6. (a) Total pair distribution functions $g(r)$ and (b) total structure factors $S(Q)$ of liquid GeTe obtained from AIMD simulations (red) and XRD measurements (black).
TABLE I. Partial coordination numbers $N_{i j}$ and total coordination number $N$ of liquid GeTe at the temperature $T$ obtained using the AIMD-simulated partial pair distribution functions. The upper integration limits applied by calculation of the partial coordination numbers are given in parentheses.

\begin{tabular}{lcccccc}
\hline \hline$T(\mathrm{~K})$ & $N_{\mathrm{GeGe}}$ & $N_{\mathrm{GeTe}}$ & $N_{\mathrm{TeTe}}$ & $N_{\mathrm{Ge} X}$ & $N_{\mathrm{Te} X}$ & $N$ \\
\hline 965 & 1.17 & 3.01 & 0.41 & 4.18 & 3.42 & 3.80 \\
& $(3.19 \AA)$ & $(3.32 \AA)$ & $(3.40 \AA)$ & & & \\
1032 & 1.21 & 2.95 & 0.48 & 4.16 & 3.43 & 3.80 \\
& $(3.18 \AA)$ & $(3.32 \AA)$ & $(3.40 \AA)$ & & & \\
1126 & 1.33 & 2.86 & 0.58 & 4.19 & 3.44 & 3.82 \\
& $(3.18 \AA)$ & $(3.34 \AA)$ & $(3.40 \AA)$ & & & \\
1259 & 1.44 & 2.80 & 0.69 & 4.24 & 3.49 & 3.87 \\
& $(3.19 \AA)$ & $(3.36 \AA)$ & $(3.40 \AA)$ & & & \\
\hline \hline
\end{tabular}

is applied to the upper limit of the integration. This cutoff is also chosen based on the studies of pure Te and Te-rich Ge-Te melts $[45,46]$. The chemical and topological short-range order exhibits notable temperature-dependent changes on the level of the partial atomic distributions, whereas the effect on the total distribution functions is practically completely hidden. Upon increasing temperature from 965 to $1259 \mathrm{~K}$, the number of $\mathrm{Ge}$-Te bonds decreases from 3 to 2.8, whereas the number of homonuclear bonds increases from 1.17 to 1.44 for $N_{\mathrm{GeGe}}$ and from 0.41 to 0.69 for $N_{\mathrm{TeTe}}$. The total coordination number $N$ exhibits a weak increase from 3.8 to 3.87 over the temperature range of $300 \mathrm{~K}$.

Analysis of the partial PDFs of liquid GeTe [Fig. 5(a)] shows that the position of the first maximum of the Ge-Ge and Ge-Te distributions does not change over the temperature range studied. This is also seen in the normalized PDFs plotted in Fig. S2 in the Supplemental Material [42]. Applying numerical differentiation to the $g_{\mathrm{GeGe}}(r)$ and $g_{\mathrm{GeTe}}(r)$ functions, the most probable bond lengths are obtained: $r_{\mathrm{GeGe}}=2.57 \AA$ and $r_{\mathrm{GeTe}}=2.74 \AA$. Numerical analysis of the range of distances corresponding to the shoulder in $g_{\mathrm{TeTe}}(r)$ yields the most probable Te-Te bond lengths $r_{\mathrm{TeTe}}$ between $2.91 \AA$ at $965 \mathrm{~K}$ and $2.98 \AA$ at $1259 \mathrm{~K}$. The Ge-Ge and Te-Te bond lengths obtained for liquid GeTe (Table II) are in good agreement with the respective values for amorphous $\operatorname{GeTe}\left(r_{\mathrm{GeGe}}=2.59 \AA\right.$ and $r_{\mathrm{GeTe}}=2.78 \AA$ ) [13]. The Te-Te bond lengths (2.91-2.98 $\AA$ ) are compatible with the $r_{\mathrm{TeTe}}$ values observed in liquid $\mathrm{Te}$

TABLE II. Bond lengths of highest probability $r_{i j}$ and mean bond lengths $\bar{r}_{i j}$ (both in angstroms) for liquid GeTe at the temperature $T$ obtained from the AIMD partial pair distribution functions. The mean bond lengths $\bar{r}_{i j}$ are calculated from the partial atomic distributions within the same limits as those applied by the calculation of the partial coordination numbers, given in Table I.

\begin{tabular}{lcccccc}
\hline \hline$T(\mathrm{~K})$ & $r_{\mathrm{GeGe}}$ & $\bar{r}_{\mathrm{GeGe}}$ & $r_{\mathrm{GeTe}}$ & $\bar{r}_{\mathrm{GeTe}}$ & $r_{\mathrm{TeTe}}$ & $\bar{r}_{\mathrm{TeTe}}$ \\
\hline 965 & 2.57 & 2.73 & 2.74 & 2.88 & 2.91 & 3.19 \\
1032 & 2.57 & 2.74 & 2.74 & 2.88 & 2.93 & 3.18 \\
1126 & 2.57 & 2.74 & 2.74 & 2.89 & 2.96 & 3.16 \\
1259 & 2.57 & 2.75 & 2.74 & 2.91 & 2.98 & 3.15 \\
\hline \hline
\end{tabular}




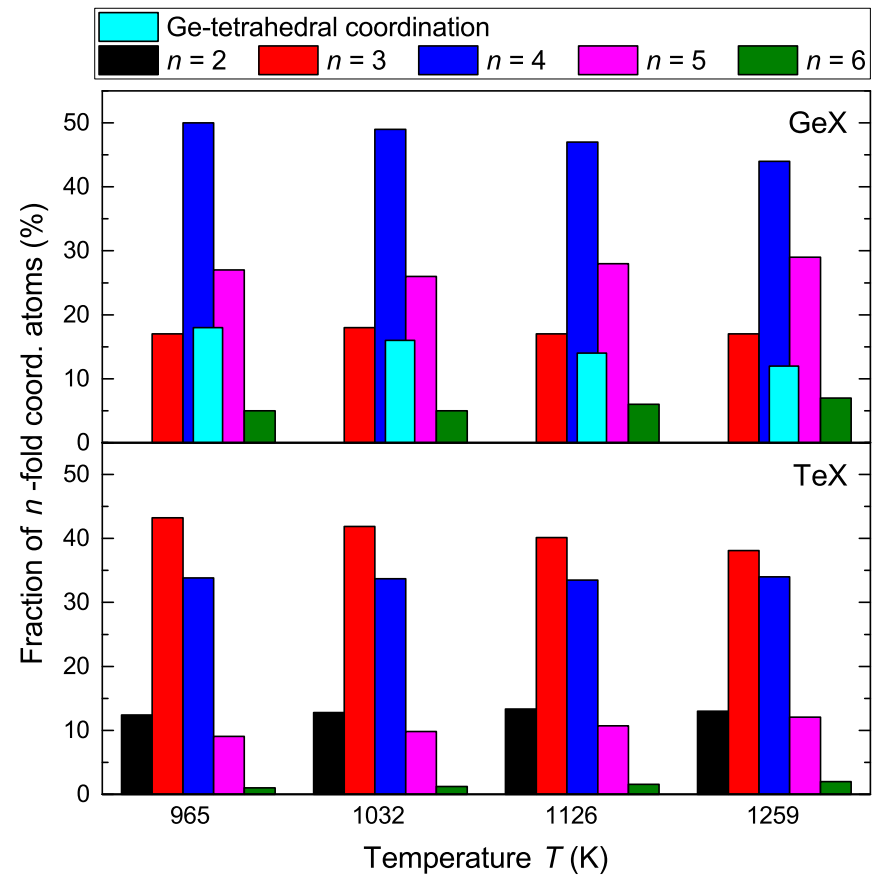

FIG. 7. Statistical distribution of $n$-fold coordinated Ge and Te atoms $(n=2,3,4,5,6)$ and $\mathrm{Ge}$ atoms in tetrahedral environment for liquid GeTe extracted from AIMD models.

$(2.89 \AA)$ and $\mathrm{Ge}_{15} \mathrm{Te}_{85}(2.83-2.88 \AA)[45,46]$. It has to be mentioned that liquid $\mathrm{Te}$ and $\mathrm{Ge}_{15} \mathrm{Te}_{85}$ alloy were studied at lower temperatures: $823 \mathrm{~K}$ [45] and up to $923 \mathrm{~K}$ [46], respectively.

It is worth mentioning that, although the most probable Ge-Ge and Ge-Te bond lengths in liquid GeTe do not vary with temperature, the asymmetry of the first peaks of the partial PDFs is enhanced with increasing temperature, as can be seen from the partial PDFs in Fig. 5(a) and from the normalized PDFs in Fig. S2 in the Supplemental Material [42]. The mean bond lengths $\bar{r}_{i j}$ (Table II) reflect the increased asymmetry at high temperature, too. Accordingly, the mean bond lengths $\bar{r}_{\mathrm{GeGe}}$ and $\bar{r}_{\mathrm{GeTe}}$ increase (albeit weakly) with temperature. On the other hand, $\bar{r}_{\mathrm{TeTe}}$ slightly decreases upon heating, opposite to the trend displayed by $r_{\mathrm{TeTe}}$. This trend stems from the fact that the shoulder around $2.9 \AA$ in the Te-Te PDFs becomes more pronounced with rising temperature [Figs. 5(a) and S2 in the Supplemental Material [42]].

To gain further insight into the local bond order in liquid $\mathrm{GeTe}$, a statistical analysis of the atomic bonds in the AIMD models is carried out (Fig. 7, and Tables S4-S6 in the Supplemental Material [42]). At $965 \mathrm{~K}$, about $50 \%$ of Ge atoms are fourfold, $27 \%$ fivefold, $17 \%$ threefold, and 5\% sixfold coordinated. As concerns the Te environment at $965 \mathrm{~K}$, most atoms are threefold $(\sim 43 \%)$ and fourfold $(\sim 34 \%)$ coordinated, although there are notable fractions of the twofold $(\sim 13 \%)$ and fivefold $(\sim 12 \%)$ coordinated Te atoms as well. The number of fourfold coordinated $\mathrm{Ge}$ and threefold coordinated Te atoms decreases with increasing temperature. This is accompanied by an increasing number of five- and sixfold coordinated Ge and Te atoms. The number of other atomic configurations remains practically unchanged over the temperature range considered.

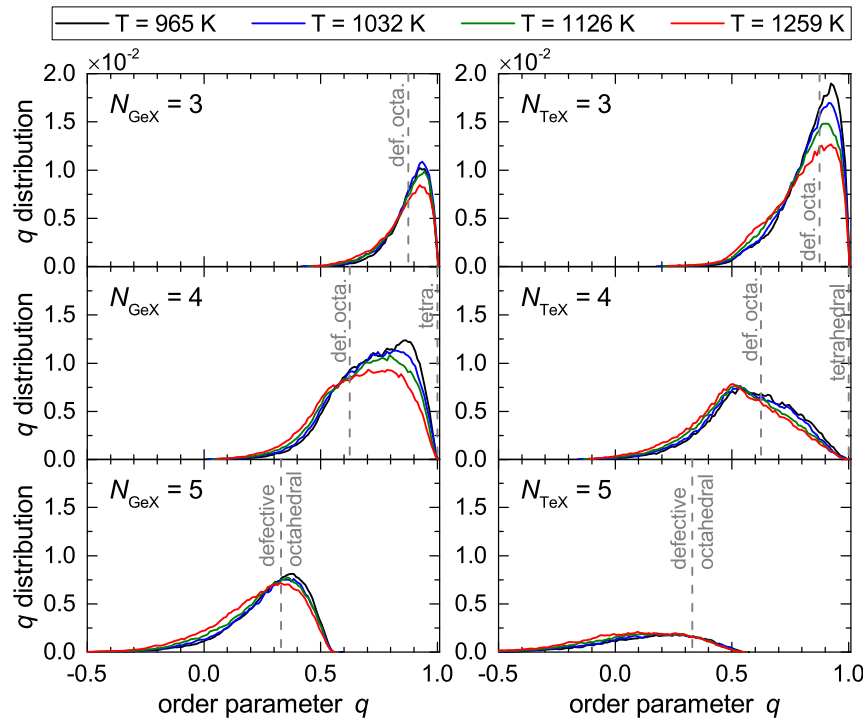

FIG. 8. Distribution of the orientational order parameter $q$ for $n$-fold coordinated $\mathrm{Ge}$ and Te atoms in liquid GeTe. Vertical dash lines mark the $q$ values corresponding to the tetrahedral $(n=4, q=$ 1) and defective octahedral $(n=3, q=7 / 8 ; n=4, q=5 / 8 ; n=$ $5, q=1 / 3$ ) geometries. The $q$ distribution for sixfold coordinated atoms is not shown because of the small number of such structures.

Our results clearly show that the structural motifs with fourfold coordinated $\mathrm{Ge}$ and threefold coordinated $\mathrm{Te}$ atoms, as well as $\mathrm{Ge}-\mathrm{Ge}$ and Te-Te homopolar bonds, found in the models of amorphous GeTe obtained by ultrafast quenching [13-15] stem from the parent liquid.

AIMD studies [13-15] have also revealed a notable amount of tetrahedrally coordinated $\mathrm{Ge}$ atoms in amorphous GeTe. To get a picture of the orientational order in liquid GeTe, we calculate the order parameter $q$ introduced by Errington and Debenedetti [47],

$$
q=q_{k}=1-\frac{3}{8} \sum_{i=1}^{N_{k}^{n b}} \sum_{j=i+1}^{N_{k}^{n b}}\left(\frac{1}{3}+\cos \theta_{i k j}\right)^{2},
$$

where $\theta_{i k j}$ is the angle formed by the lines connecting an atom $k$ and its nearest neighbors $i$ and $j$. The summation runs over all pairs $(i, j)$ of atoms $i, j=1, \ldots, N_{k}^{n b}$ bound to atom $k$. The $q$-parameter distributions for the $n$-fold coordinated Ge and Te atoms, with $n=3,4$ and 5, are plotted in Fig. 8. Due to their small quantities, distributions for sixfold coordinated $\mathrm{Ge}$ and Te atoms are not displayed in Fig. 8. The value of $q=1$ corresponds to a perfect tetrahedral structure. Here the fraction of tetrahedral Ge atoms is calculated by integrating the $q$ distributions from $q=0.8$ to 1.0 , as has been done for amorphous GeTe in Ref. [13]. The defective octahedral environments are represented by values of $q$ equal to $7 / 8$, $5 / 8$, and $1 / 3$ for three-, four- and fivefold coordination, respectively. A value of $q=0$ corresponds to perfect sixfold octahedral structures. The $q$ distributions (Fig. 8) indicate that Ge atoms are mainly in tetrahedral, defective octahedral, and octahedral sites (the latter distribution is not shown, as mentioned above). The number of tetrahedral structures increases with decreasing temperature of the liquid. 


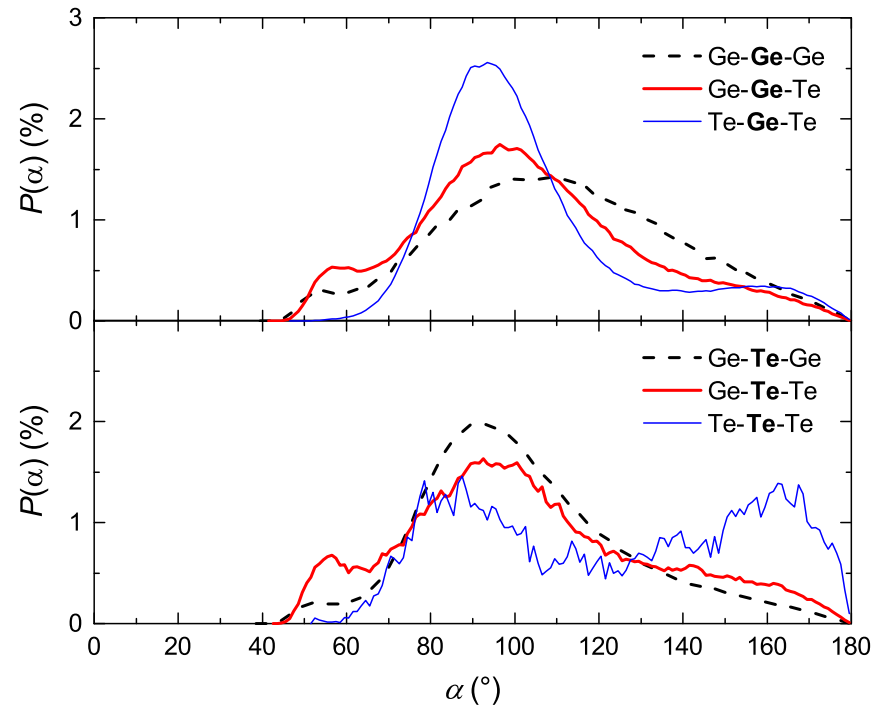

FIG. 9. Bond-angle distributions for Ge and Te atoms in liquid $\mathrm{GeTe}$ at $965 \mathrm{~K}$. The distributions for all temperatures considered $(965,1032,1126,1259 \mathrm{~K})$ are shown in Fig. S3 in the Supplemental Material [42].

Raty et al. [15] ascribed the formation of tetrahedral structures in amorphous GeTe to the presence of $\mathrm{Ge}-\mathrm{Ge}$ bonds.
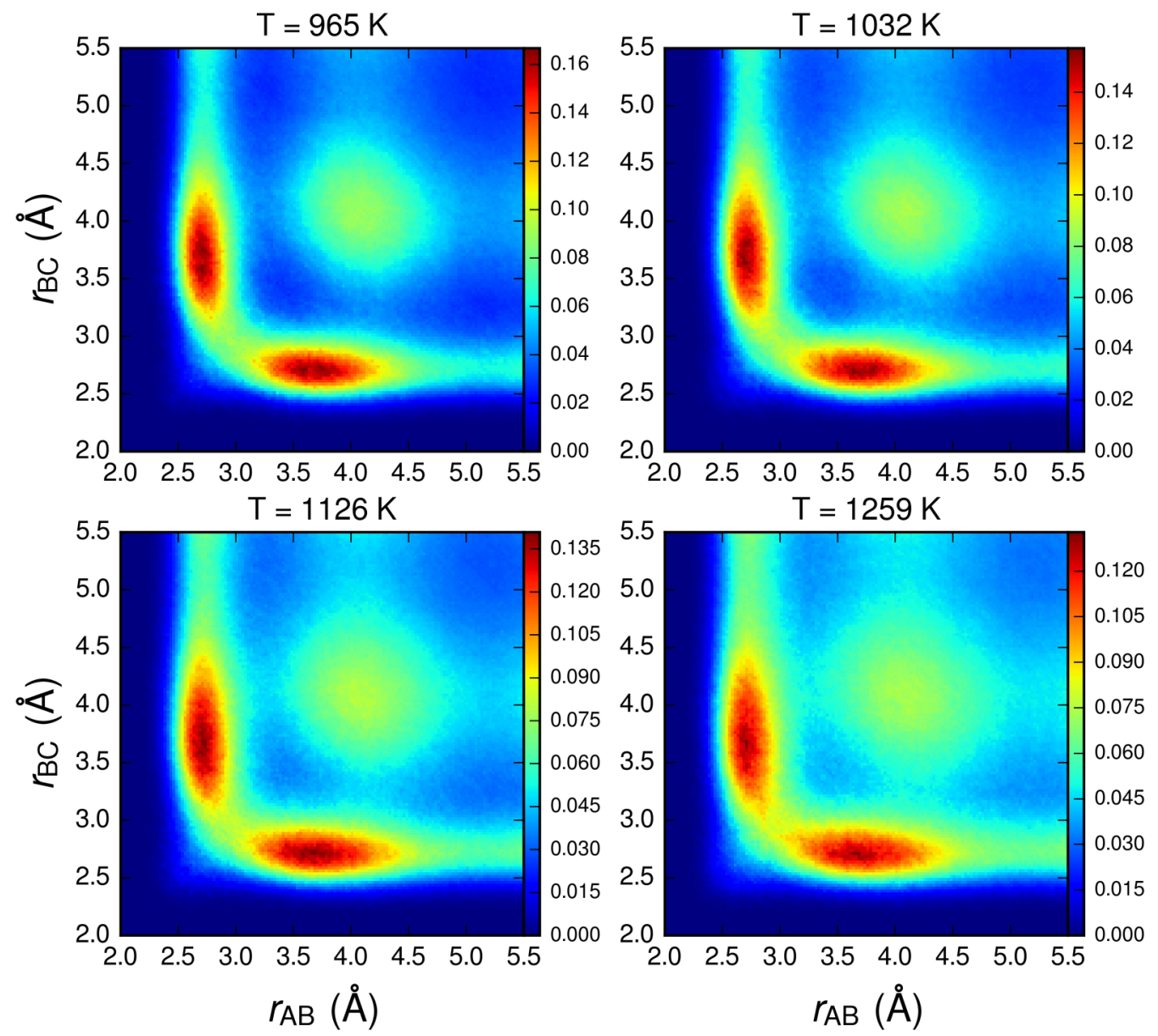

FIG. 10. Total angular-limited three-body correlation (ALTBC) for liquid GeTe at 965, 1032, 1126, and 1259 K. Two well-defined peaks outside the diagonal $\left(r_{\mathrm{AB}}=r_{\mathrm{BC}}\right)$ indicate Peierls-like distortion in the liquid state, which weakly decreases with increasing temperature.
Such bonds are relatively abundant in the liquid state (Table I, and Table S4 in the Supplemental Material [42]) and thus found in the amorphous state obtained by ultrafast quenching as well. Analysis of the Ge-centered tetrahedra with respect to the number of homopolar bonds they incorporate (Table S6 in the Supplemental Material [42]) shows that more than $80 \%$ of these tetrahedra contain at least one Ge-Ge bond. The majority of the tetrahedra contains one or two Ge-Ge bonds: $44 \%$ and $35 \%$ at $965 \mathrm{~K}$, respectively. The number of $\mathrm{Ge}-\mathrm{Ge}$ bonds in liquid GeTe becomes smaller with decreasing temperature (Table I, and Table S4 in the Supplemental Material [42]), in agreement with the fact that they are energetically unfavorable [15], whereas the fraction of tetrahedral structures with (at least) one Ge-Ge bond increases (Table S6 in the Supplemental Material [42]). Therefore, we attribute the increase in Gecentered tetrahedra as well as fourfold coordinated Ge atoms upon cooling to the decrease of thermal disorder.

The atomic configurations in liquid GeTe following from the orientational order parameter are also represented in the bond-angle distributions (BADs) resolved for $\mathrm{Ge}$ and Te central atoms (Fig. 9 for $965 \mathrm{~K}$, and Fig. S3 in the Supplemental Material [42] for all temperatures considered). The Te-Ge-Te, Ge-Te-Te, Ge-Te-Ge, and Ge-Ge-Te BADs exhibit maxima close to $90^{\circ}$ (at about $93^{\circ}, 95^{\circ}, 90^{\circ}$, and $94^{\circ}$, respectively), whereas the $\mathrm{Ge}-\mathrm{Ge}-\mathrm{Ge} \mathrm{BAD}$ is characterized by a broad distribution with a peak at about $109^{\circ}$, indicating 

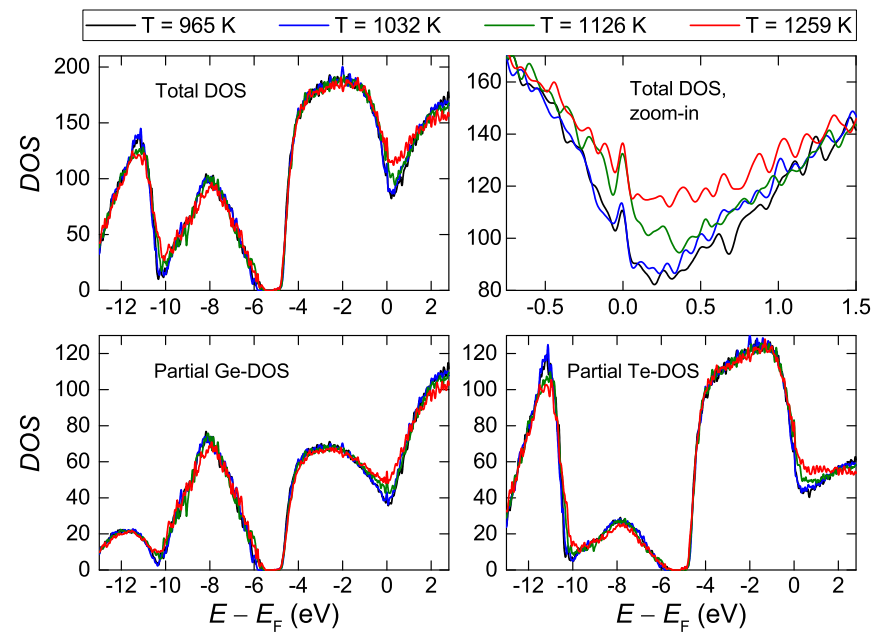

FIG. 11. Total and partial electronic density of states (DOS) for liquid GeTe at $965,1032,1126$, and $1259 \mathrm{~K}$. A zoom-in of the total DOS in the upper right panel elucidates the temperature dependence of DOS close to the Fermi energy $E_{\mathrm{F}}$. Projections of the DOS onto the single orbitals of Ge and Te atoms are shown in Fig. S5 in the Supplemental Material [42]).

octahedral and tetrahedral coordination, respectively. The $\mathrm{Te}-\mathrm{Te}-\mathrm{Te}$ distribution shows two peaks at about $86^{\circ}$ and $165^{\circ}$, both in agreement with octahedral coordination but the second additionally indicative of aligned Te chains. However, it needs to be stressed that the appearance of pure, bound Te triplets is a relatively rare event in liquid GeTe and their contribution is therefore less significant.

To shed further light on the evolution of the liquid state with increasing temperature, the angular-limited three-body correlations (ALTBCs) (Fig. 10) are calculated from the AIMD trajectories (partial ALTBC distributions are shown in Figs. S4(a)-S4(d) in the Supplemental Material [42]). The ALTBC distribution function gives the probability of finding a triplet of atoms having one bond of length $r_{1}$ almost aligned with a second bond of length $r_{2}$. Here, we record atomic triplets with interbond angles up to a deviation of $25^{\circ}$. The ALTBC plots exhibit two peaks outside the diagonal $\left(r_{\mathrm{AB}}=r_{\mathrm{BC}}\right)$, which correspond to alternating, aligned short and long bonds. This feature provides evidence for Peierls-like distortion [21] in liquid GeTe as reported first by Raty et al. [20]. The distortion in liquid $\mathrm{GeTe}$ decreases with increasing temperature, but rather weakly in the temperature range considered (Fig. 10). A more pronounced decrease of Peierls-like distortion has been observed in liquid $\mathrm{Ge}_{2} \mathrm{Sb}_{2} \mathrm{Te}_{5}$ [24].

The calculated total electronic density of states (DOS) of liquid GeTe and its contributions from $\mathrm{Ge}$ and $\mathrm{Te}$ atoms (Fig. 11) display a pseudogap at the Fermi energy $E_{\mathrm{F}}$. The plots of the density of states projected onto the $s, p$, and $d$ orbitals (Fig. S5 in the Supplemental Material [42]) show that the DOS has predominant $p$ character near $E_{\mathrm{F}}$, whereas the $s$ orbitals mostly contribute to the states below $-6 \mathrm{eV}$. At 965 and $1032 \mathrm{~K}$, the DOS in the pseudogap reaches about half its maximum value, pointing to the existence of electron localized states in accordance to the criterion of Mott and Allgaier [48]. In agreement with the negative temperature dependence of
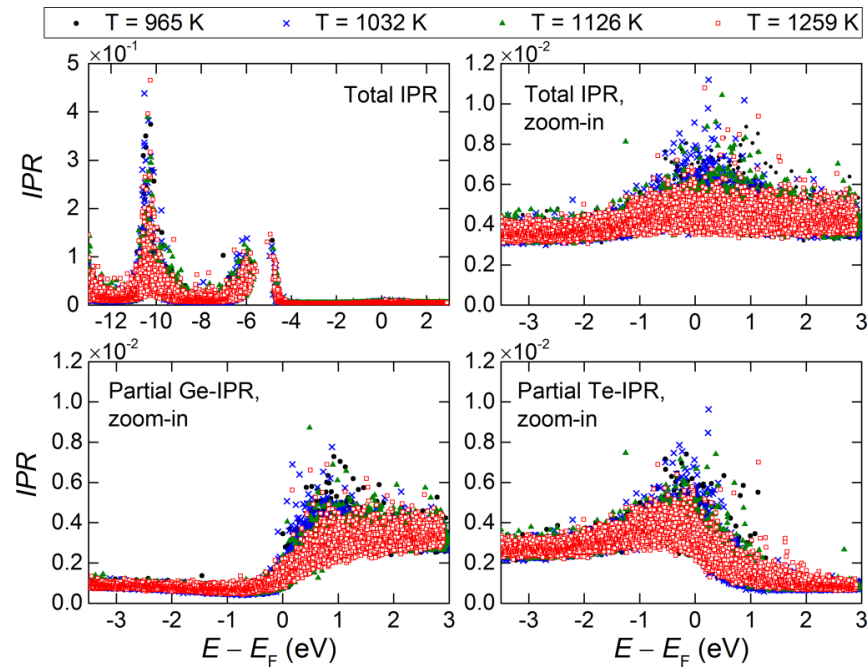

FIG. 12. The inverse participation ratio IPR, Eq. (6), for liquid $\mathrm{GeTe}$ at different temperatures as a function of energy. The zoom-in plots show the total and partial IPR near the Fermi energy $E_{\mathrm{F}}$. Higher values of the IPR at the Fermi level indicate electron localization, which is decreasing upon increasing temperature. The shapes of the partial IPR curves in the proximity of $E_{\mathrm{F}}$ are different: While the $\mathrm{Ge}$ IPR increases in the energy range slightly above the Fermi level, the Te IPR decreases in the same range.

the electrical resistivity reported for liquid GeTe [17], the pseudogap decreases with increasing temperature.

To estimate the degree of localization of the electron states, we calculate the inverse participation ratio (IPR) $[49,50]$, defined as

$$
\operatorname{IPR}\left(\left|\psi_{\mathrm{i}}\right\rangle\right)=\frac{\sum_{j=1}^{N}\left|\Phi_{j}^{(i)}\right|^{4}}{\left(\sum_{j=1}^{N}\left|\Phi_{j}^{(i)}\right|^{2}\right)^{2}},
$$

with

$$
\left|\Phi_{j}^{(i)}\right|^{2}=\sum_{k=1}^{N} \sum_{\mu \in A_{j}} \sum_{\nu \in A_{k}} c_{\mu}^{(i) *} S_{\mu \nu} c_{\nu}^{(i)} .
$$

The quantity $\Phi_{j}^{(i)}$ is the amplitude at atom $j$ of the eigenstate $\left|\psi_{i}\right\rangle$ with energy $\varepsilon_{i}$ and the eigenstates $\left|\psi_{i}\right\rangle=\sum_{\mu} c_{\mu}^{(i)}\left|\varphi_{\mu}\right\rangle$ in our calculations are expanded in terms of Gaussian atomic orbitals $\left|\varphi_{\mu}\right\rangle . S_{\mu \nu}=\left\langle\varphi_{\mu} \mid \varphi_{\nu}\right\rangle$ denotes the atomic overlap matrix, and $A_{j}$ represents a set of orbitals corresponding to atom $j$. The IPR is roughly equal to the inverse of the number of atoms on which the eigenstate is localized. In case of an infinitely large system, the IPR takes a finite value for localized states, and it is zero for any extended eigenstate. In a sufficiently large but finite supercell, the IPR is significantly larger for localized states than for extended ones. In our AIMD models of liquid $\mathrm{GeTe}$, the IPR of the electronic states at 965 and $1032 \mathrm{~K}$ shows increased values near $E_{\mathrm{F}}$ and becomes less pronounced with increasing temperature (Fig. 12). This is consistent with the negative $\mathrm{d} \rho / \mathrm{d} T$ indicating an increasing mean free path in liquid GeTe upon heating. The result for $\mathrm{GeTe}$ is in line with the electronic properties of liquid $\mathrm{Ge}_{2} \mathrm{Sb}_{2} \mathrm{Te}_{5}$ obtained in our earlier work [24] and reproduced for the sake of comparison in Figs. S6 and S7 in the Supplemental Material [42]. The DOS 
and IPR graphs for liquid $\mathrm{Ge}_{2} \mathrm{Sb}_{2} \mathrm{Te}_{5}$ exhibit the shape and behavior similar to those for liquid GeTe.

The self-diffusion coefficients of liquid $\mathrm{GeTe}$ at 969 , 1028,1128 , and $1257 \mathrm{~K}$, determined from the mean square displacements obtained by MD modeling as well as the corresponding dynamic viscosities calculated using the StokesEinstein relation are given in the Supplemental Material [Figs. S8(a)-S8(d)] [42]. For a better statistics for these values, the length of the MD trajectories is increased to $120 \mathrm{ps,}$ which explains slightly different temperatures compared to the structural data. The dynamic viscosity of liquid GeTe obtained from the AIMD simulations shows a good correlation with the measured data plotted in Fig. 1. The fit of the theoretical viscosity with the Arrhenius equation yields $E_{a}=0.313 \mathrm{eV}$ and $\eta_{0}=0.082 \mathrm{mPa}$. The activation energy is slightly larger than the experimental value, while the prefactors are similar. This observation is contrary to the case of liquid $\mathrm{Ge}_{2} \mathrm{Sb}_{2} \mathrm{Te}_{5}$, for which the theoretical "vdW-DF2" activation energy and prefactor have been shown to be, respectively, slightly smaller and twice as large as the experimental ones [24]. However, in both sets of simulations the theoretical $E_{\mathrm{a}}$ and $\eta_{0}$ are affected by statistical uncertainties due to the small number of data points. For the sake of comparison, in Fig. 1 we also include the viscosity values obtained by Sosso et al. using an accurate neural-network $(\mathrm{NN})$ potential [43] fitted to structures obtained from a large set of DFT simulations which have been based on the generalized-gradient approximation (GGA). The inclusion of the nonlocal correlations as implemented in the vdW-DF2 functional appears to lead to a more viscous state, yielding better agreement with experiments. Nonetheless, we should point out that our results are affected by finite size effects, due to which the viscosities are significantly overestimated. Proper assessment of these effects would require the use of larger models, which is computationally challenging and expensive. Correction of the finite size errors could lead to an even better match with experimental data. This point is further discussed in the next section.

\section{CONCLUDING REMARKS}

In this work, we experimentally investigate the structural properties, the molar volume, and the dynamic viscosity of liquid $\mathrm{GeTe}$ as a function of temperature. The experiments are complemented with AIMD and RMC simulations. In the AIMD simulations, we employ the vdW-DF2 functional, which provides a good description of van der Waals interactions by including nonlocal correlations. Our simulations indicate that this functional yields a more viscous liquid state of GeTe as compared to GGA functionals, against which the neural-network potential used by Sosso et al. [43] has been fitted. This result is in line with the study of liquid $\mathrm{Ge}_{15} \mathrm{Te}_{85}$ by Micoulaut [51], where it has been shown that $\mathrm{vdW}$ Grimme corrections to GGA functionals reduce the diffusion coefficients. However, it is important to stress that our values of the viscosities are overestimated due to the relatively small size of the supercell, which contains 512 atoms. Owing to the periodic boundary conditions, the slowly decaying hydrodynamic flow created by a diffusing particle causes an interference between the particle and its periodic images [52]. As a consequence, the resulting diffusivities are smaller than the correct values obtained in the limit of very large systems, leading to overestimated viscosities. Nevertheless, since the values we obtain are more than three times larger than the ones of Sosso et al. [43], we expect that correction for this systematic error will not change the trend. Quite the contrary, such correction could even improve the agreement with experimental data.

Both experimental measurements and AIMD calculations carried out in the present work show that the viscosity of GeTe in the equilibrium and weakly supercooled liquid states obeys the Arrhenius law (Fig. 1) and is characterized by a small activation energy of the order of $0.3 \mathrm{eV}$. Similar values of $E_{\mathrm{a}}$ ( 0.23 and $0.31 \mathrm{eV}$, depending on the fitting algorithm) were obtained by Sosso et al. [43] for GeTe in the supercooled liquid state between $T_{\mathrm{m}}=998 \mathrm{~K}$ and $700 \mathrm{~K}$. Fitting their viscosities, calculated using the Stokes-Einstein relation [Eq. (2)], in the temperature range between 998 and $500 \mathrm{~K}$ (open squares in Fig. 1) yields $\eta_{0}=0.052 \mathrm{mPa} \mathrm{s}, E_{a}=0.244 \mathrm{eV}$. Such a low activation energy is indicative of a high fragility of liquid GeTe.

Computing the dynamic viscosity by scaling the diffusion coefficient with the size of the simulation cell (Sosso et al. [43]) showed that the Stokes-Einstein relation breaks down at the temperature below $700 \mathrm{~K}$ and dynamic viscosity steeply increases by aproaching the glass transition at $T_{g}=423 \mathrm{~K}$ [53] (see full squares in Fig. 1). Unfortunately, there is no experimental data on the viscosity of highly supercooled liquid GeTe. Experimental evidence for the high fragility of the related compound $\mathrm{Ge}_{2} \mathrm{Sb}_{2} \mathrm{Te}_{5}$ has been provided by Orava et al. [54]. Due to this property, the activation energy for the viscosity becomes much larger at low temperatures, which results in a very stable amorphous state. Whether the high fragility is a generic property of phase-change alloys (PCAs) or not is an open question. In particular, the low-temperature kinetic properties of another important family of PCAs, the doped SbTe compounds, are currently debated [55-58].

\section{ACKNOWLEDGMENTS}

This work has been financially supported by the German Research Foundation DFG (Contracts No. Ka-3209/6-1 and No. Ma-5339/2-1). We also acknowledge the computational resources granted by JARA-HPC from RWTH Aachen University under Projects No. JARA0089 and No. JARA0150, as well as funding by the DFG within the collaborative research center SFB 917 "Nanoswitches.” P.J. was supported by NKFIH (National Research, Development and Innovation Office) Grant No. SNN 116198. High-temperature XRD measurements were performed at the light source PETRA III at DESY, Hamburg, a member of the Helmholtz Association (HGF). We thank O. Shuleshova for help in using the electromagnetic levitation facility and U. Rütt and $\mathrm{O}$. Gutowski for support at the beamline P07. Also, high-temperature XRD experiments were performed at the European Synchrotron Radiation Facility (ESRF), Grenoble, France. We are grateful to J. Wright for providing assistance in using the beamline ID11 and data treatment. The neutron diffraction experiments at LLB were supported by the European Commission through the Access Activities of the Integrated Infrastructure Initiative for Neutron Scattering and Muon Spectroscopy (NMI3), supported by the European Commission under the 7th Framework Programme 
through the Key Action: Strengthening the European Research Area, Research Infrastructures, Contract No. NMI3-II/FP7 283883. We thank L. Cormier, L. Hennet, and J. Darpentigny for support during neutron diffraction experiments and afterwards.

H.W. and M.S. contributed equally to this work.
[1] N. Yamada, E. Ohno, K. Nishiuchi, N. Akahira, and M. Takao, J. Appl. Phys. 69, 2849 (1991).

[2] Phase Change Materials and Applications, edited by S. Raoux and M. Wuttig (Springer, Berlin, 2008).

[3] M. Chen, K. A. Rubin, and R. W. Barton, Appl. Phys. Lett. 49, 502 (1986).

[4] S. Raoux, W. Welnic, and D. Ielmini, Chem. Rev. 110, 240 (2010).

[5] W. Zhang, V. L. Deringer, R. Dronskowski, R. Mazzarello, E. Ma, and M. Wuttig, MRS Bull. 40, 856 (2015).

[6] J. S. Meena, S. M. Sze, U. Chand, and T. Y. Tseng, Nanoscale Res. Lett. 9, 526 (2014).

[7] G. Bruns, P. Merkelbach, C. Schlockermann, M. Salinga, M. Wuttig, T. D. Happ, J. B. Philipp, and M. Kund, Appl. Phys. Lett. 95, 043108 (2009).

[8] J. Chattopadhyay, T. Boucherle, and H. Von Schnering, J. Phys. C: Solid State Phys. 20, 1431 (1987).

[9] K. M. Rabe and J. D. Joannopoulos, Phys. Rev. B 36, 6631 (1987).

[10] R. Shaltaf, E. Durgun, J.-Y. Raty, P. Ghosez, and X. Gonze, Phys. Rev. B 78, 205203 (2008).

[11] D. Di Sante, P. Barone, R. Bertacco, and S. Picozzi, Adv. Mater. 25, 509 (2013).

[12] J. Akola and R. O. Jones, Phys. Rev. B 76, 235201 (2007).

[13] R. Mazzarello, S. Caravati, S. Angioletti-Uberti, M. Bernasconi, and M. Parrinello, Phys. Rev. Lett. 104, 085503 (2010); 107, 039902(E) (2011).

[14] V. L. Deringer, W. Zhang, M. Lumeij, S. Maintz, M. Wuttig, R. Mazzarello, and R. Dronskowski, Angew. Chem., Int. Ed. 53, 10817 (2014).

[15] J.-Y. Raty, W. Zhang, J. Luckas, C. Chen, R. Mazzarello, C. Bichara, and M. Wuttig, Nat. Commun. 6, 7467 (2015).

[16] A. A. Piarristeguy, M. Micoulaut, R. Escalier, P. Jóvári, I. Kaban, J. van Eijk, J. Luckas, S. Ravindren, P. Boolchand, and A. Pradel, J. Chem. Phys. 143, 074502 (2015).

[17] H. U. Tschirner, I. P. Pazdrij, and R. Weikart, Wiss. Z. d. Tech. Hochsch. Karl-Marx-Stadt. 25, 219 (1983).

[18] Y. Tsuchiya, J. Phys. Soc. Jpn. 60, 227 (1991).

[19] F. Herwig and M. Wobst, Z. Metallkd. 82, 917 (1991).

[20] J.-Y. Raty, V. Godlevsky, Ph. Ghosez, C. Bichara, J. P. Gaspard, and J. R. Chelikowsky, Phys. Rev. Lett. 85, 1950 (2000).

[21] J.-P. Gaspard, A. Pellegatti, F. Marinelli, and C. Bichara, Philos. Mag. B 77, 727 (1998).

[22] J. Akola and R. O. Jones, Phys. Rev. Lett. 100, 205502 (2008).

[23] K. Lee, E. D. Murray, L. Kong, B. I. Lundqvist, and D. C. Langreth, Phys. Rev. B 82, 081101 (2010).

[24] M. Schumacher, H. Weber, P. Jóvári, Y. Tsuchiya, T. G. A. Youngs, I. Kaban, and R. Mazzarello, Sci. Rep. 6, 27434 (2016).

[25] O. Shuleshova, I. Kaban, S. Ziller, and U. Reinhold (unpublished); https://www.ifw-dresden.de/institutes/ikm/researchteams-and-topics/solidification-processes-and-complexstructures/facilities/.
[26] G. Ashiotis, A. Deschildre, Z. Nawaz, J. P. Wright, D. Karkoulis, F. E. Piccac, and J. Kieffer, J. Appl. Crystallogr. 48, 510 (2015).

[27] A. P. Hammersley, S. O. Svensson, M. Hanfland, A. N. Fitch, and D. Häusermann, High Press. Res. 14, 235 (1996).

[28] H. H. M. Balyuzi, Acta Crystallogr., Sect. A 31, 600 (1975).

[29] T. E. Faber and J. M. Ziman, Philos. Mag. 11, 153 (1965).

[30] D. Waasmaier and A. Kirfel, Acta Crystallogr., Sect. A 51, 416 (1995).

[31] I. A. Blech and B. L. Averbach, Phys. Rev. 137, A1113 (1965).

[32] H. H. Paalman and J. C. Pings, J. Appl. Phys. 33, 2635 (1962).

[33] V. F. Sears, Neutron News 3, 26 (1992).

[34] Y. Tscuchiya, J. Phys. Soc. Jpn. 57, 3851 (1988).

[35] S. Gruner and W. Hoyer, J. Alloys Compd. 480, 629 (2009).

[36] R. Roscoe and W. Bainbridge, Proc. Phys. Soc., London 72, 576 (1958).

[37] R. F. Brooks, A. T. Dinsdale, and P. N. Quested, Meas. Sci. Technol. 16, 354 (2005).

[38] J. VandeVondele, M. Krack, F. Mohamed, M. Parrinello, T. Chassaing, and J. Hutter, Comput. Phys. Commun. 167, 103 (2005).

[39] S. Goedecker, N. Teter, and J. Hutter, Phys. Rev. B 54, 1703 (1996).

[40] T. Kühne, M. Krack, F. R. Mohamed, and M. Parrinello, Phys. Rev. Lett. 98, 066401 (2007).

[41] T. Kühne, M. Krack, and M. Parrinello, J. Chem. Theory. Comput. 5, 235 (2009).

[42] See Supplemental Material at http://link.aps.org/supplemental/ 10.1103/PhysRevB.96.054204 for additional data on liquid GeTe obtained by experiments and AIMD simulations: measured values of the molar volume, number density, and dynamic viscosity, dimensions of the AIMD simulation cells, normalized partial pair distribution functions, coordination statistics and bond angle distributions for $\mathrm{Ge}$ and $\mathrm{Te}$ atoms, electronic properties of liquid $\mathrm{GeTe}$ and $\mathrm{Ge}_{2} \mathrm{Sb}_{2} \mathrm{Te}_{5}$, mean square displacements of the particles, and diffusion coefficients (Tables S1-S6 and Figs. S1-S8).

[43] G. C. Sosso, J. Behler, and M. Bernasconi, Phys. Status Solidi B 249, 1880 (2012); 250, 1453 (2013).

[44] O. Gereben, P. Jóvári, L. Temleitner, and L. Pusztai, J. Optoelectron. Adv. Mater. 9, 3021 (2007).

[45] W. Hoyer, I. Kaban, and T. Halm, J. Optoelectron. Adv. Mater. 3, 255 (2001).

[46] I. Kaban, P. Jóvári, W. Hoyer, R. G. Delaplane, and A. Wannberg, J. Phys.: Condens. Matter 18, 2749 (2006).

[47] J. R. Errington and P. G. Debenedetti, Nature 409, 318 (2001).

[48] N. F. Mott and R. S. Allgaier, Phys. Status Solidi 21, 343 (1967).

[49] F. Wegner, Z. Phys. B: Condens. Matter 36, 209 (1980).

[50] D. J. Thouless, Phys. Rep. 13, 93 (1974).

[51] M. Micoulaut, J. Chem. Phys. 138, 061103 (2013). 
[52] I.-C. Yeh and G. Hummer, J. Phys. Chem. B 108, 15873 (2004).

[53] M. Chen and K. A. Rubin, Proc. SPIE 1078, 150 (1989).

[54] J. Orava, A. L. Greer, B. Gholipour, D. W. Hewak, and C. E. Smith, Nat. Mater. 11, 279 (2012).

[55] M. Salinga, E. Carria, A. Kaldenbach, M. Bornhöfft, J. Benke, J. Mayer, and M. Wuttig, Nat. Commun. 4, 2371 (2013).
[56] W. Zhang, I. Ronneberger, P. Zalden, M. Xu, M. Salinga, M. Wuttig, and R. Mazzarello, Sci. Rep. 4, 6529 (2014).

[57] J. Orava, D. W. Hewak, and A. L. Greer, Adv. Funct. Mater. 25, 4851 (2015).

[58] J. Orava, H. Weber, I. Kaban, and A. L. Greer, J. Chem. Phys. 144, 194503 (2016). 